University of Windsor

Scholarship at UWindsor

Industrial and Manufacturing Systems

Department of Industrial and Manufacturing

Engineering Publications

Systems Engineering

7-15-2016

\title{
Optimal disposition decisions for a remanufacturing system considering time value of products
}

\author{
Mostafa Pazoki \\ University of Windsor \\ Walid Abdul-Kader \\ University of Windsor
}

Follow this and additional works at: https://scholar.uwindsor.ca/industrialengpub

Part of the Industrial Engineering Commons

\section{Recommended Citation}

Pazoki, Mostafa and Abdul-Kader, Walid. (2016). Optimal disposition decisions for a remanufacturing system considering time value of products. Computers and Industrial Engineering, 99, 124-136.

https://scholar.uwindsor.ca/industrialengpub/12

This Article is brought to you for free and open access by the Department of Industrial and Manufacturing Systems Engineering at Scholarship at UWindsor. It has been accepted for inclusion in Industrial and Manufacturing Systems Engineering Publications by an authorized administrator of Scholarship at UWindsor. For more information, please contact scholarship@uwindsor.ca. 


\section{Optimal disposition decisions for a remanufacturing system considering time value of products}

\section{Mostafa Pazoki}

pazoki@uwindsor.ca

Walid Abdul-Kader ${ }^{\mathrm{b}, *}$

kader@uwindsor.ca

a Industrial and Manufacturing Systems Engineering, University of Windsor, Canada

bechanical, Automotive and Materials Engineering, University of Windsor, Canada

${ }^{*}$ Corresponding author

\section{Abstract}

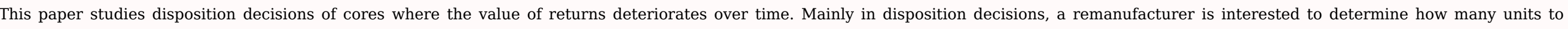

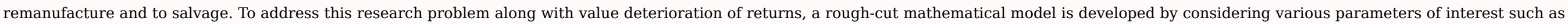

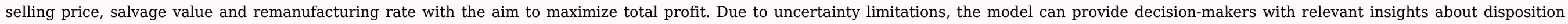

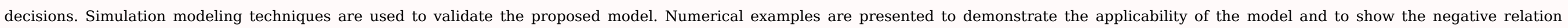
between the deterioration rate and the total profit. However, the above-indicated parameters of selling price, salvage value and remanufacturing rate work the opposite way.

Keywords: Remanufacturing; Time value of products; Mathematical model; Simulation

\section{Introduction}

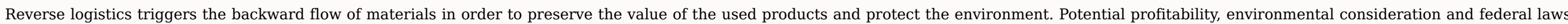

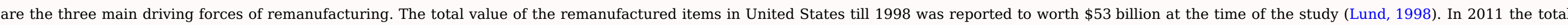
value of the remanufacture production went up to $\$ 43$ billion, expecting to grow each year. ${ }^{1}$

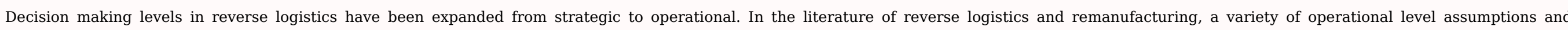

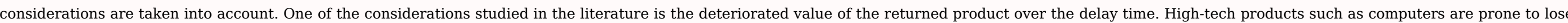

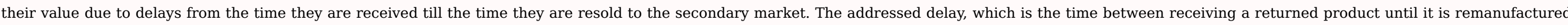

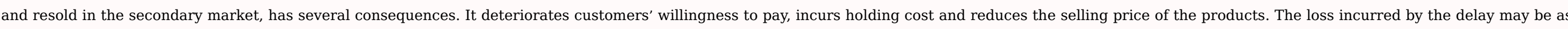

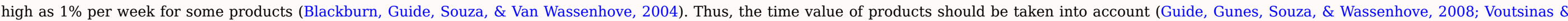

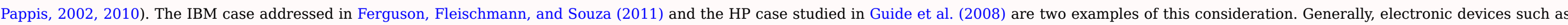

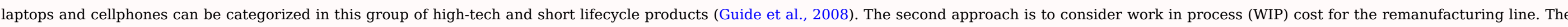

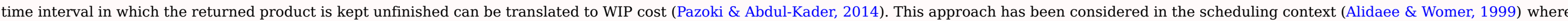
the value of products depends on the completion time (Janiak \& Krysiak, 2007).

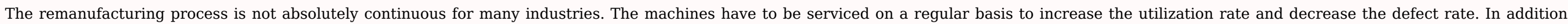

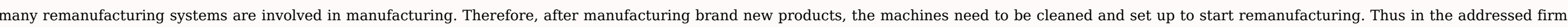

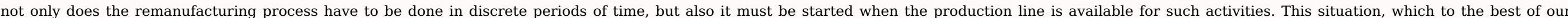




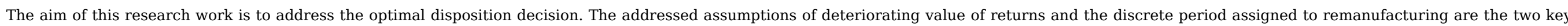

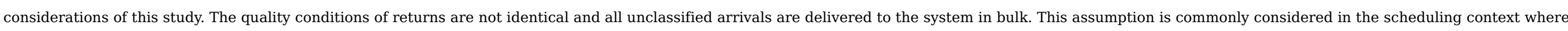

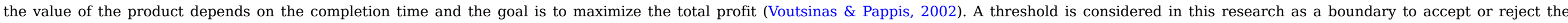

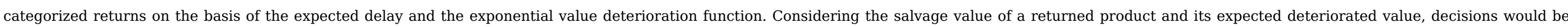

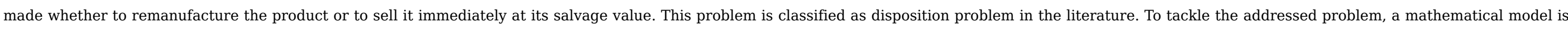
presented. The schematic view of the disposition decision is illustrated in Fig. 1.

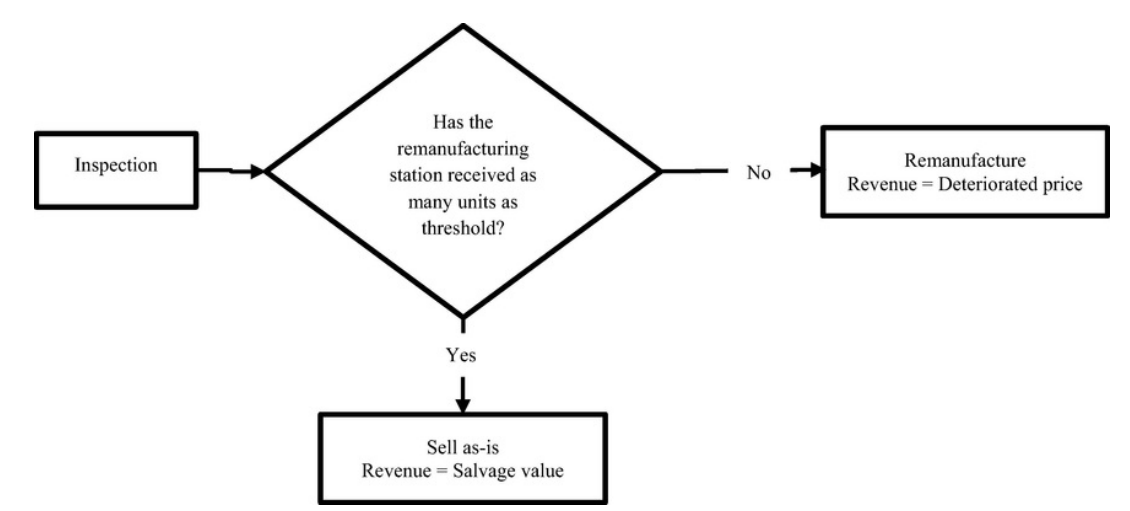

Fig. 1 Disposition decision and flow of materials.

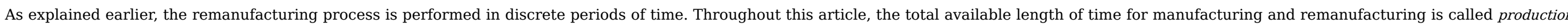

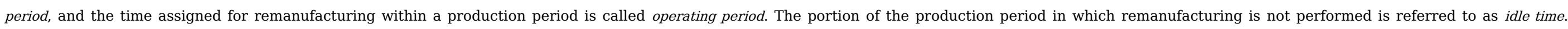

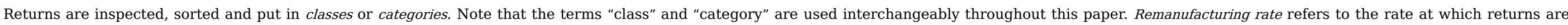
remanufactured, and remanufacturing time is the time it takes to remanufacture a unit of product (inverse of remanufacturing rate).

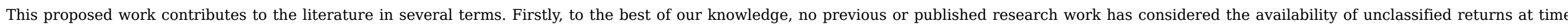

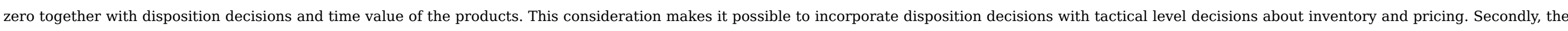

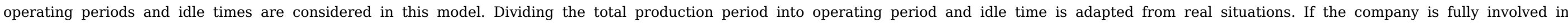

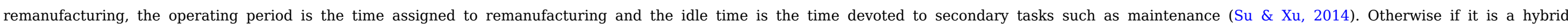

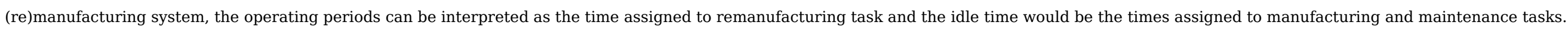

Thus, as a summary, this proposed research contributes to the literature by considering (in addition to the quantity to remanufacture and to salvage) the following points:

- Deterioration value for returns and time value of the products]

- Assuming availability of returns at time zero, and.

- Availability of discrete time intervals for remanufacturing.

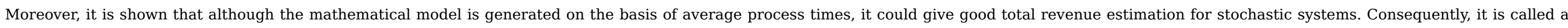
rough-cut model.

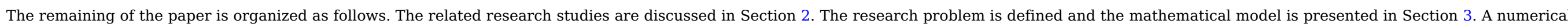




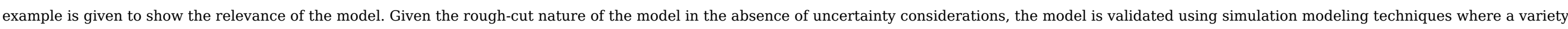

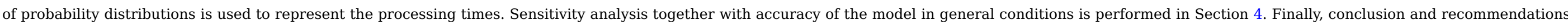
for future research are presented.

\section{Literature review}

\subsection{On the quality variability, marginal value of time and arrival/service times}

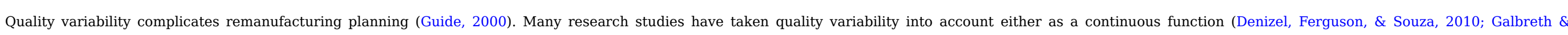

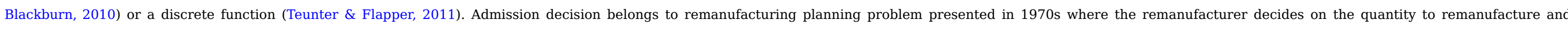

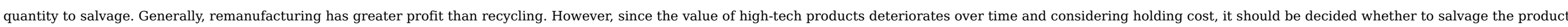

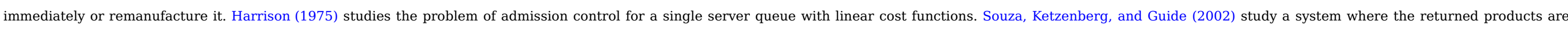

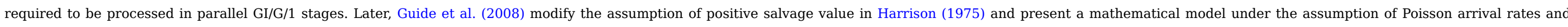

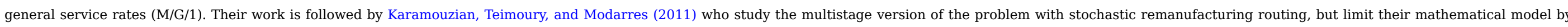

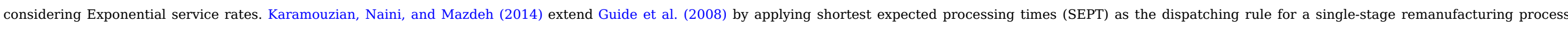

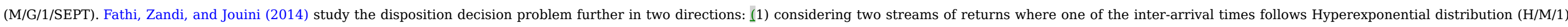

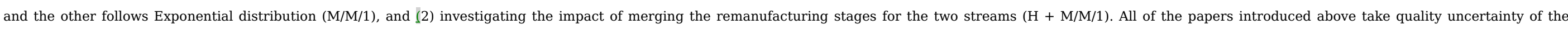
returns, exponentially deteriorated values and net present value into account.

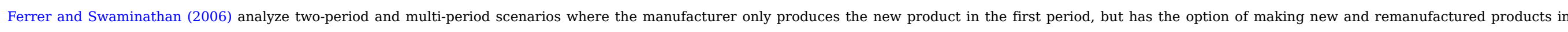

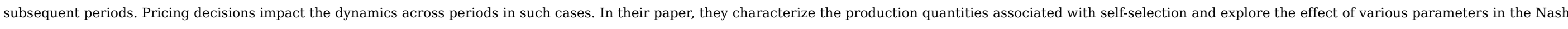

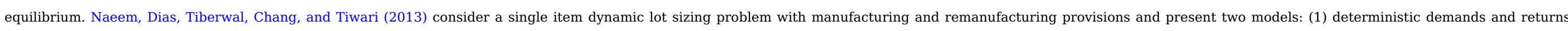

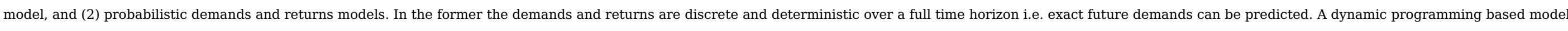

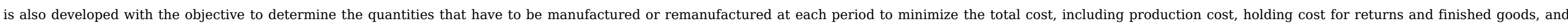

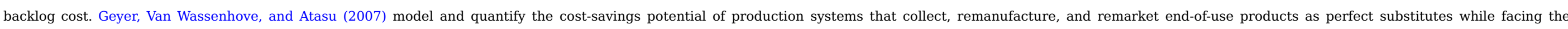

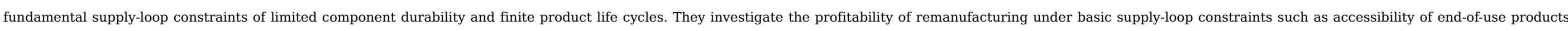
(collection rate), technical feasibility of remanufacturing (durability), and market demand for remanufactured products (life cycle).

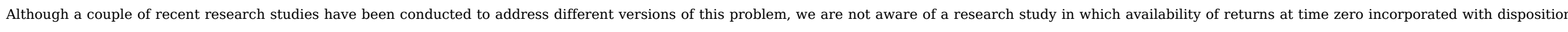

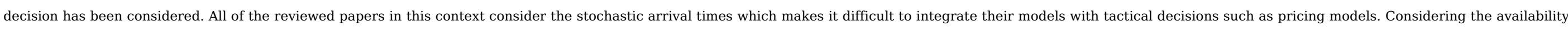
of all the jobs at time zero is common in scheduling context (Alidaee \& Womer, 1999). Thus, this paper contributes to the current literature on disposition decision in remanufacturing problem.

\subsection{On the modeling methodology}

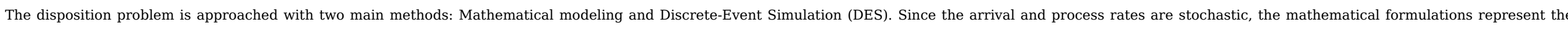

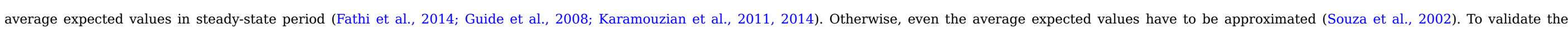

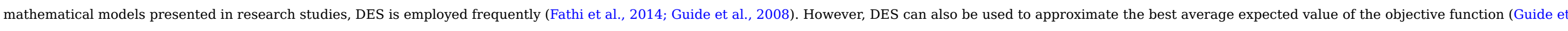

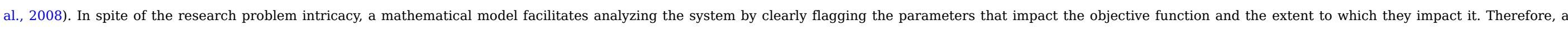
mathematical model is an appropriate method to tackle this problem.

\section{Problem definition and mathematical model development}

\subsection{Problem definition}




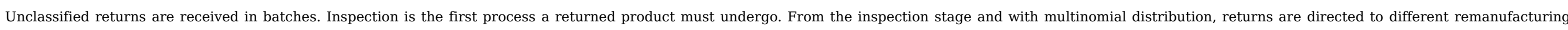

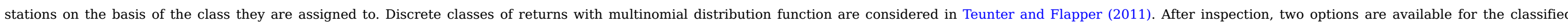

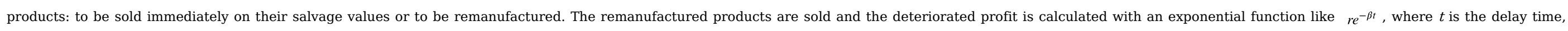

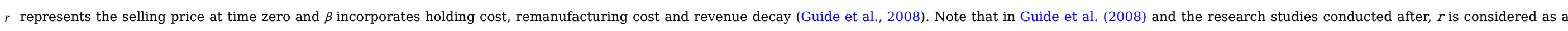

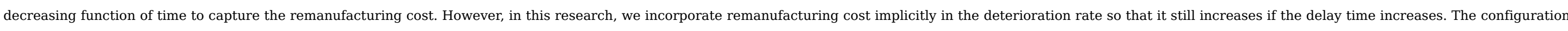

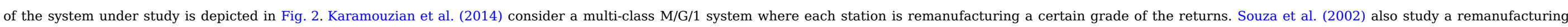
system consisting of multi-class GI/G/1 remanufacturing station where each station is devoted to a certain quality category or type of the returns.

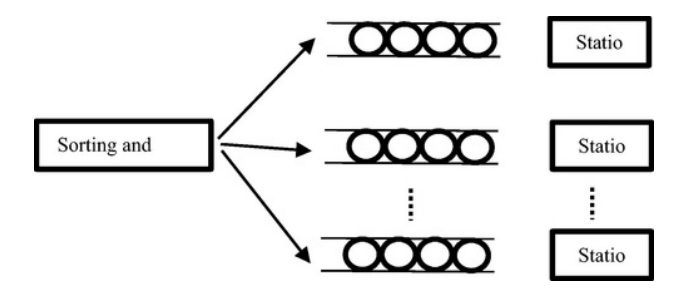

Fig. 2 Material flow in the system under study.

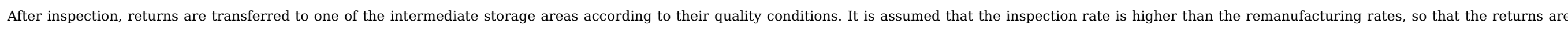

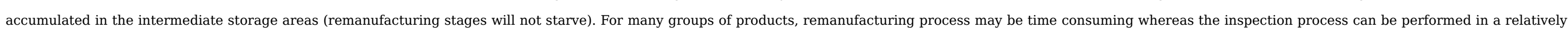
shorter period of time. Therefore, the addressed assumption makes sense for many real world cases.

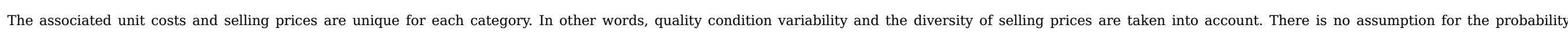
distributions of inspection and remanufacturing times; only the expected values are considered.

\subsection{Mathematical model}

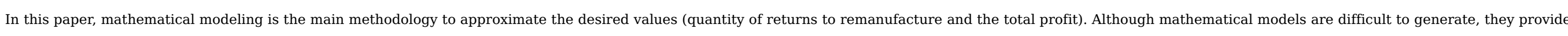

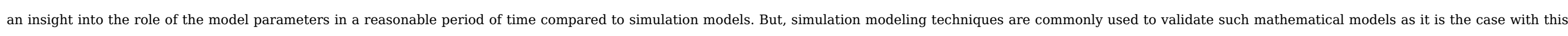
proposed model.

The notation, parameters and decision variables used in the model are as follows:

I: Set of quality condition classes and remanufacturing stations.

$S_{i}$ : The number of returns to be remanufactured during one operating period from class $i \in I$.

$R_{i}$ : The number of operating periods required to remanufacture all of the returns for class $i \in I$

$R_{i}^{j}$ : The operating period in which the $\pi$ th return belonging to class $i \in I$ would be remanufactured.

$W_{i}^{j}$ : The waiting/delay time of the $j$ th item belonging to the th class.

$\beta:$ The deterioration rate.

$\mu_{i}$ : The rate at which station $i \in I$ remanufactures the returns (remanufacturing rate).

$p_{i}$ : The probability that a return belongs to class $i \in I$.

$\lambda$ : Inspection rate. 
Sal: The salvage value of a return which is sent for recycling.

T: Production period.

U: Operating period.

$\Psi:$ The total number of received returns.

$k_{i}$ : The quantity of returns to remanufacture from class $i \in I$.

$Z_{i}$ : The total deteriorated profit gained by remanufacturing or salvaging the returns categorized in class $i \in I$. However, the total profit from all the items is: $Z=\sum_{i \in I} Z_{i}$

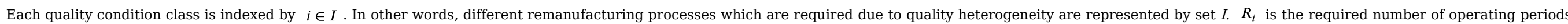

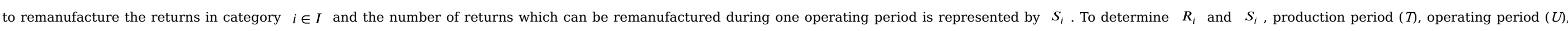

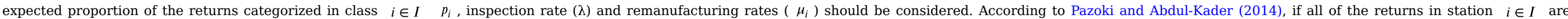
remanufactured continuously with no breaks or idle times, the delay time of the $j$ th item, $W_{i}^{j}$, would be:

$W_{i}^{j}=\frac{j}{\mu_{i}}+\frac{1}{\lambda p_{i}}$

Thus, the number of returns to remanufacture during each operating period (except the last period), $S_{i}$, is (Pazoki \& Abdul-Kader, 2014): $S_{i}=\left\lfloor\mu_{i}\left(U-1 / \lambda p_{i}\right)\right\rfloor$ $S_{i}=\left\lfloor\mu_{i} U\right\rfloor$

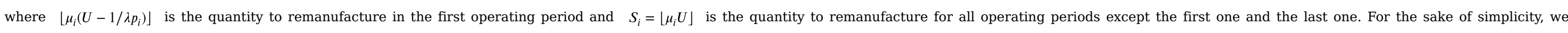

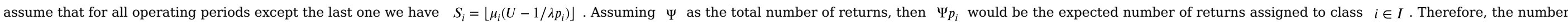
of required operating periods to remanufacture the returns assigned to class $i \in I$ is (if all of the returns are supposed to be remanufactured):

In a similar way, the operating period order at which the $t$ th returned item would be remanufactured can be calculated as indicated below:

$$
R_{i}^{j}=\left\lceil\frac{j}{S_{i}}\right\rceil
$$

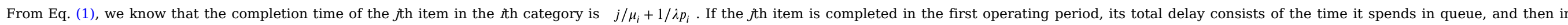

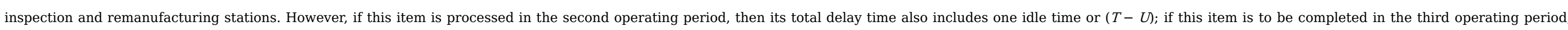

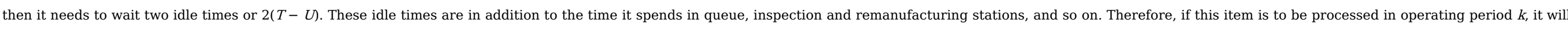
see $(k-1)$ idle times or $(k-1)(T-U)$, before it is finished. Thus, incorporating Eqs. (1) and (4), the delay time of the $j$ th returned item considering operating periods and idle times should be revised to:

$$
W_{i}^{j}=(T-U)\left(R_{i}^{j}-1\right)+\frac{j}{\mu_{i}}+\frac{1}{\lambda p_{i}}
$$

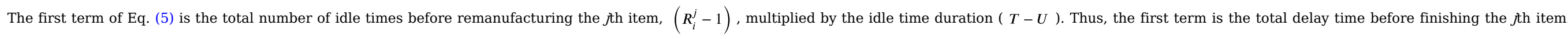

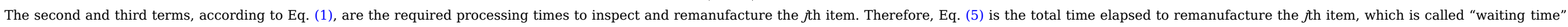
Eqs. (1)-(5) are the basic equations upon which the mathematical model is built.

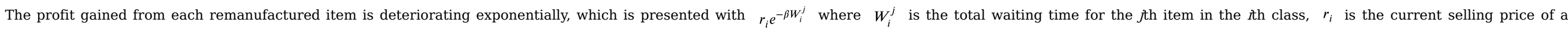


The total deteriorated profit for remanufacturing the returns from class $i \in I$ in the first operating period is calculated in Eq. (6).

$$
\sum_{n=1}^{n=S_{i}} r_{i} e^{-\beta\left(\frac{1}{\lambda p_{i}}+\frac{n}{\mu_{i}}\right)}=r_{i} e^{-\beta\left(\frac{1}{\lambda p_{i}}+\frac{1}{\mu_{i}}\right)}\left(\frac{1-e^{-\frac{S_{i} \beta}{\mu_{i}}}}{1-e^{-\frac{\beta}{\mu_{i}}}}\right)
$$

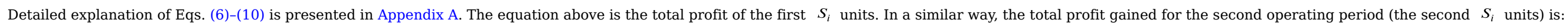

$$
\sum_{n=S_{i}+1}^{n=2 S_{i}} r_{i} e^{-\beta\left(T-U+\frac{1}{\lambda p_{i}}+\frac{n}{\mu_{i}}\right)}=r_{i} e^{-\beta\left(T-U+\frac{1}{\lambda p_{i}}+\frac{S_{i}+1}{\mu_{i}}\right)}\left(\frac{1-e^{-\frac{S_{i} \beta}{\mu_{i}}}}{1-e^{-\frac{\beta}{\mu_{i}}}}\right)
$$

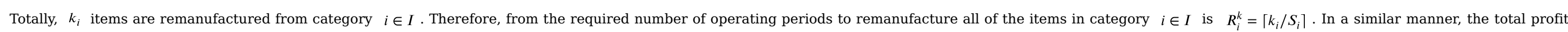
for the operating period $R_{i}^{k}-1$ is:

$$
\left.\sum_{n=\left(R_{i}^{k}-2\right) S_{i}+1}^{n=\left(R_{i}^{k}-1\right) S_{i}} r_{i} e^{-\beta\left(\left(R_{i}^{k}-2\right)(T-U)+\frac{1}{\lambda p_{i}}+\frac{n}{\mu_{i}}\right)}=r_{i} e^{-\beta\left(\left(R_{i}^{k}-2\right)(T-U)+\frac{1}{\lambda p_{i}}+\frac{\left(R_{i}^{k}-2\right) S_{i}+1}{\mu_{i}}\right.}\right)\left(\frac{1-e^{-\frac{S_{i} \beta}{\mu_{i}}}}{1-e^{-\frac{\beta}{\mu_{i}}}}\right)
$$

Thus, the total remanufacturing profit gained during first $R_{i}^{k}-1$ operating periods can be obtained by Eq. (9).

$$
\sum_{n=1}^{n=R_{i}^{k}-1} r_{i} e^{-\beta\left((n-1)(T-U)+\frac{1}{\lambda_{i}}+\frac{(n-1) S_{i}+1}{\mu_{i}}\right)}\left(\frac{1-e^{-\frac{S_{i} \beta}{\mu_{i}}}}{1-e^{-\frac{\beta}{\mu_{i}}}}\right)=r_{i} e^{-\beta\left(\frac{1}{\lambda_{i}}+\frac{1}{\mu_{i}}\right)}\left(\frac{1-e^{-\beta \frac{S_{i}}{\mu_{i}}}}{1-e^{-\beta \frac{1}{\mu_{i}}}}\right)\left(\frac{1-e^{-\beta\left(R_{i}^{k}-1\right)\left(T-U+\frac{S_{i}}{\mu_{i}}\right)}}{1-e^{-\beta\left(T-U+\frac{S_{i}}{\mu_{i}}\right)}}\right)
$$

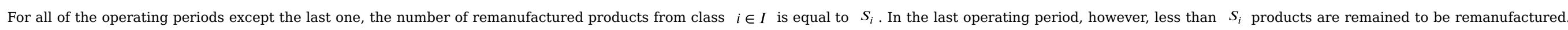

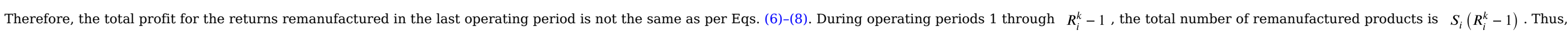

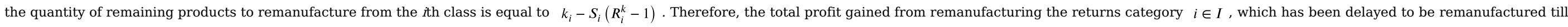
the last operating period is:

$\sum_{n=S_{i}\left(R_{i}^{k}-1\right)+1}^{n=k_{i}} r_{i} e^{\left(-\beta(T-U)\left(R_{i}^{k}-1\right)+\frac{1}{\lambda p_{i}}+\frac{n}{\mu_{i}}\right)}=r_{i} e^{\left(-\beta(T-U)\left(R_{i}^{k}-1\right)+\frac{1}{\lambda p_{i}}+\frac{1}{\mu_{i}}\right)}\left(\frac{e^{-\beta \frac{S_{i}\left(R_{i}^{k}-1\right)}{\mu_{i}}}-e^{-\beta \frac{k_{i}}{\mu_{i}}}}{1-e^{-\frac{\beta}{\mu_{i}}}}\right)$

The total remanufacturing profit function of the ith class (see Eq. (11) below) is obtained by adding (10) and (9).

$$
Z_{i}=r_{i}\left(e^{\left(-\beta(T-U)\left(R_{i}^{k}-1\right)+\frac{1}{\lambda p_{i}}+\frac{1}{\mu_{i}}\right)}\left(\frac{e^{-\beta \frac{S_{i}\left(R_{i}^{k}-1\right)}{\mu_{i}}}-e^{-\beta \frac{k_{i}}{\mu_{i}}}}{1-e^{-\frac{\beta}{\mu_{i}}}}\right)+e^{-\beta\left(\frac{1}{\lambda p_{i}}+\frac{1}{\mu_{i}}\right)}\left(\frac{1-e^{-\beta \frac{S_{i}}{\mu_{i}}}}{1-e^{-\beta \frac{1}{\mu_{i}}}}\right)\left(\frac{1-e^{-\beta\left(R_{i}^{k}-1\right)\left(T-U+\frac{S_{i}}{\mu_{i}}\right)}}{1-e^{-\beta\left(T-U+\frac{S_{i}}{\mu_{i}}\right)}}\right)\right)
$$

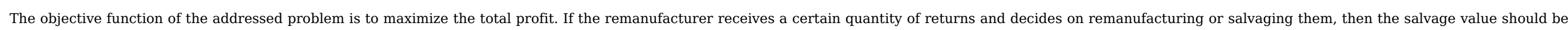
included in the total profit function. Hence, the mathematical model would be:

$$
\text { Maximize : } \quad Z=\sum_{i \in I} r_{i}\left(e^{\left(-\beta(T-U)\left(R_{i}^{k}-1\right)+\frac{1}{p_{p}}+\frac{1}{\mu_{i}}\right)}\left(\frac{e^{-\beta \frac{S_{i}\left(R_{i}^{k}-1\right)}{\mu_{i}}}-e^{-\beta \frac{k_{i}}{\mu_{i}}}}{1-e^{-\frac{\beta}{\mu_{i}}}}\right)+e^{-\beta\left(\frac{1}{\bar{p}_{i}}+\frac{1}{\mu_{i}}\right)}\left(\frac{1-e^{-\beta \frac{S_{i}}{\mu_{i}}}}{1-e^{-\beta \frac{1}{\mu_{i}}}}\right)\left(\frac{1-e^{-\beta\left(R_{i}^{k}-1\right)\left(T-U+\frac{S_{i}}{\mu_{i}}\right)}}{1-e^{-\beta\left(T-U+\frac{S_{i}}{\mu_{i}}\right.}}\right)\right)+S a l\left(\Psi-\sum_{i \in I} k_{i}\right)
$$

Subject to:

$k_{i} \leqslant \Psi p_{i}, \quad \forall i \in I$

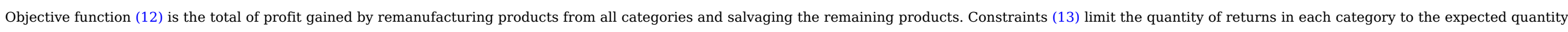




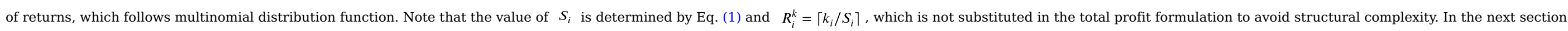

\section{a numerical example is presented and the results are discussed.}

\subsection{Mathematical model verification}

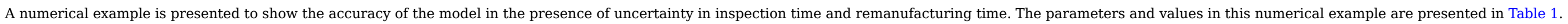

Table 1 Parameters used in the numerical example.

\begin{tabular}{|c|c|}
\hline Parameters and notation & 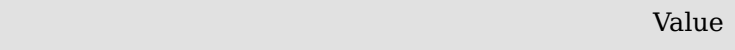 \\
\hline Number of stations (i) & 4 \\
\hline Time unit & $t$ \\
\hline$p_{i}$ & $0.1,0.2,0.3,0.4$ \\
\hline$r_{i}$ & $1000,1250,1500,1800$ \\
\hline$\lambda$ & 700 per $t$ \\
\hline$\mu_{i}$ & $70,80,90,100$ per $t$ \\
\hline$\beta$ & 0.05 per $t$ \\
\hline$\Psi$ & 10,000 \\
\hline$T$ & $24 t$ \\
\hline$U$ & $8 t$ \\
\hline
\end{tabular}

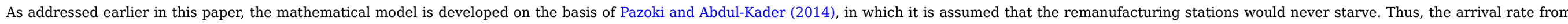

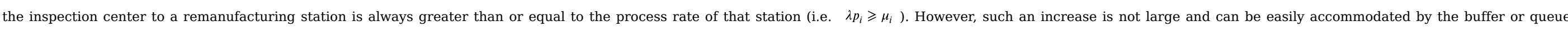
located upstream of the station.

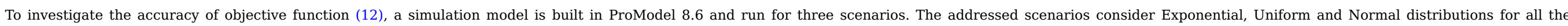

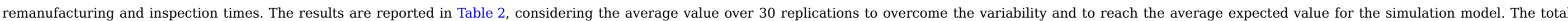
profit value obtained by the mathematical model is $4,129,022$. The relative error is calculated as follows:

|Total profit of scenario - Total profit of mathematical model|/Total profit of scenario.

Table 2 Total profit for the deterministic $(4,129,022)$ and stochastic systems.

\begin{tabular}{|c|c|c|c|c|}
\hline Scenarios & Inspection time & Remanufacturing time & Total profit & Relative error compared to the mathematical model \\
\hline 1 & $E(1 / \lambda)$ & $E\left(1 / \mu_{i}\right)$ & $4,065,982$ & 0.016 \\
\hline 2 & $U(1 / 2 \lambda, 3 / 2 \lambda)$ & $U\left(1 / 2 \mu_{i}, 3 / 2 \mu_{i}\right)$ & $4,105,114$ & 0.006 \\
\hline 3 & $N(1 / \lambda, 1 / 2 \lambda)$ & $N\left(1 / \mu_{i}, 1 / 2 \mu_{i}\right)$ & $4,086,683$ & 0.010 \\
\hline
\end{tabular}




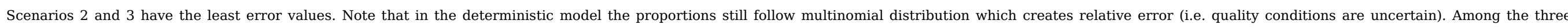

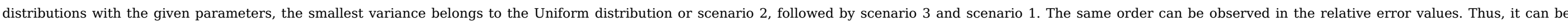

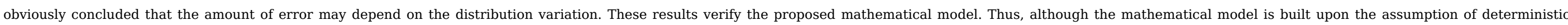
process times, we expect to see the same average results in the case of stochastic process times.

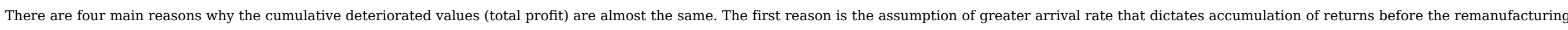

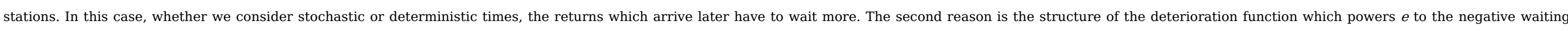

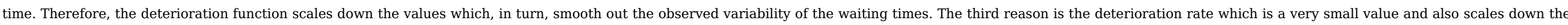

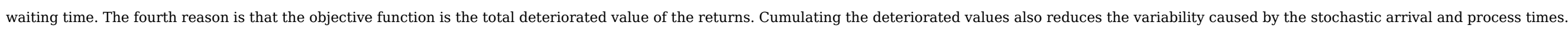

In this section, we showed that the mathematical model is relatively acceptable for stochastic systems. In the next section, we further investigate the model performance in the presence of uncertainty.

\subsection{Brief notes about model complexity}

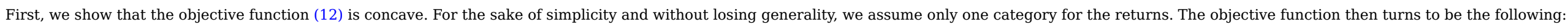

$$
\text { Maximize : } \left.\quad Z=r\left(e^{\left(-\beta(T-U)\left(R^{k}-1\right)+\frac{1}{\lambda p}+\frac{1}{\mu}\right)}\left(\frac{e^{-\beta \frac{S\left(R^{k}-1\right)}{\mu}}-e^{-\beta \frac{k}{\mu}}}{1-e^{-\frac{\beta}{\mu}}}\right)+e^{-\beta\left(\frac{1}{\lambda p}+\frac{1}{\mu}\right.}\right)\left(\frac{1-e^{-\beta \frac{S}{\mu}}}{1-e^{-\beta \frac{1}{\mu}}}\right)\left(\frac{1-e^{-\beta\left(R^{k}-1\right)\left(T-U+\frac{S}{\mu}\right)}}{1-e^{-\beta\left(T-U+\frac{S}{\mu}\right)}}\right)\right)+S a l(\Psi-k)
$$

Assuming $R^{k}=k / S$ and $S=\mu U$, the first order derivative of $\mathrm{Z}$ is:

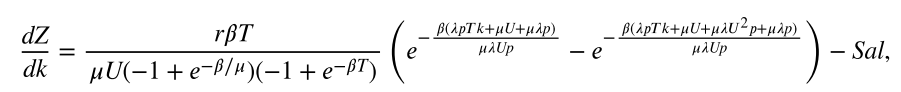

and the second order derivative is:

$$
\frac{d^{2} Z}{d k^{2}}=-\frac{r \beta^{2} T^{2}}{\mu^{2} U^{2}\left(-1+e^{-\beta / \mu}\right)\left(-1+e^{-\beta T}\right)}\left(e^{-\frac{\beta(\lambda p T k+\mu U U+\mu \lambda p)}{\mu \lambda U p}}-e^{-\frac{\beta\left(\lambda p T k+\mu U+\mu \lambda U^{2} p+\mu \lambda \hat{\lambda} p\right)}{\mu \lambda U_{p}}}\right) .
$$

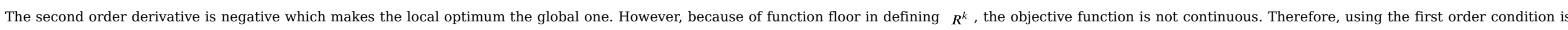

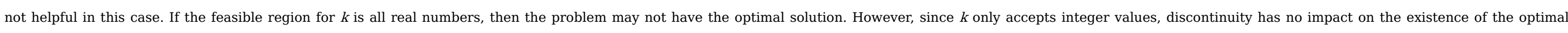

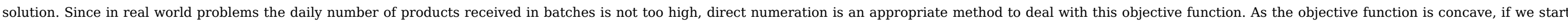

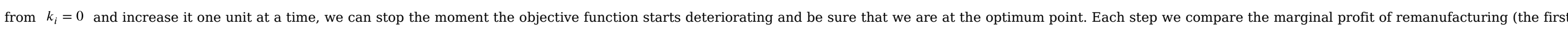

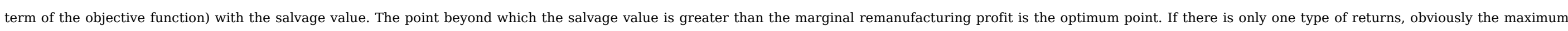

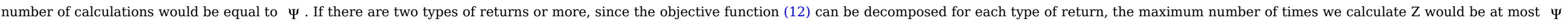
Hence, the computational burden is not significant for this model.

\section{Numerical experiments and factor analysis}

\subsection{Investigating the impact of remanufacturing rates}

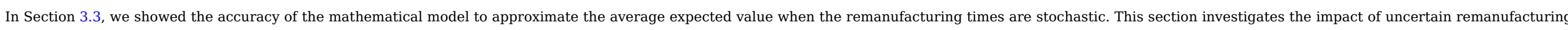

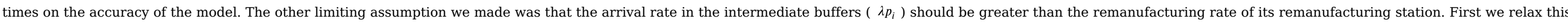
assumption and calculate the accuracy of the mathematical model. Note that in the presence of uncertainty, the simulation model has been run for 30 replications to obtain the average expected profit.

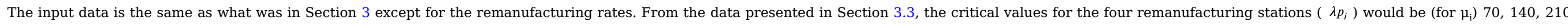

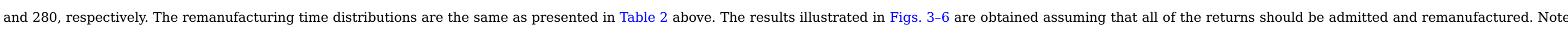

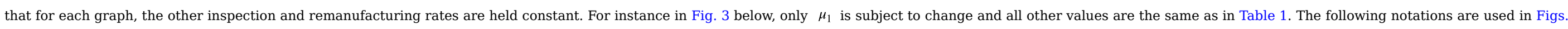


- Exponential is the objective function values obtained by the simulation model for exponentially distributed inspection and remanufacturing times.

- Uniform is the objective function values obtained by the simulation model for uniformly distributed inspection and remanufacturing times.

- Normal is the objective function values obtained by the simulation model for normally distributed inspection and remanufacturing times.

- Mathematical model is the objective function value obtained by the mathematical model solved in MAPLE 16.

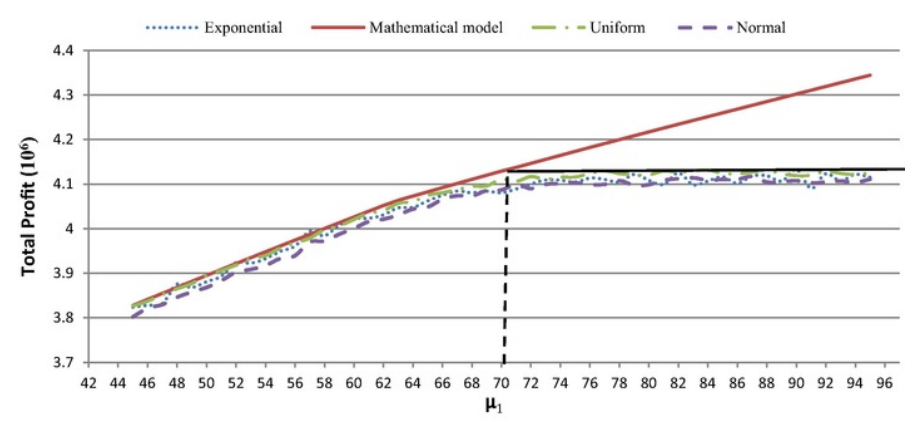

Fig. 3 Impact of the first station's remanufacturing rate, $\mu_{1}$, on the objective function for $\mu_{2}=80, \mu_{3}=90, \mu_{4}=100$, and $\lambda=700$.

The results are reported in Tables 4-7. The graphs below are depicting the impacts.

Table 3 Parameters used in the numerical experiments.

\begin{tabular}{|c|c|}
\hline Parameters and notation & Value \\
\hline Number of stations $(i)$ & 1 \\
\hline Time unit & $\mathrm{h}$ \\
\hline$p_{i}$ & 1 \\
\hline$r_{i}$ & $\$ 8000$ \\
\hline$\lambda$ & 3.12 per hour \\
\hline$\mu_{i}$ & 1.04 per hour \\
\hline$\beta$ & 0.0006875 per hour \\
\hline$\Psi$ & 250 or 500 items \\
\hline$T$ & $24 \mathrm{~h}$ \\
\hline$U$ & $24 \mathrm{~h}$ \\
\hline
\end{tabular}

Table 4 Scenario definition. 


\begin{tabular}{|c|c|c|c|c|c|c|}
\hline 1 & High & Medium & Low & High & Medium & Low \\
\hline 2 & Medium & Medium & Medium & Medium & Medium & Medium \\
\hline 3 & High & Medium & Low & Medium & Medium & Medium \\
\hline 4 & Low & Medium & High & High & Medium & Low \\
\hline 5 & Medium & Medium & Medium & Low & Medium & High \\
\hline
\end{tabular}

Table 5 Input data for numerical analysis.

\begin{tabular}{|c|c|c|}
\hline Parameters & Levels & Value \\
\hline \multirow[t]{3}{*}{$r_{i}$} & High & 1000 (unit) \\
\hline & Medium & 750 (unit) \\
\hline & Low & 500 (unit) \\
\hline \multirow[t]{3}{*}{$\mu_{i}$} & High & 100 (unit/t) \\
\hline & Medium & 75 (unit/t) \\
\hline & Low & 50 (unit/t) \\
\hline \multirow[t]{4}{*}{$\beta$} & \multirow[t]{4}{*}{ N/A } & $0.0005(\$ / t)$ \\
\hline & & $0.001(\$ / t)$ \\
\hline & & $0.002(\$ / t)$ \\
\hline & & $0.003(\$ / t)$ \\
\hline$\lambda p_{i}$ & N/A & 200 (unit/t) \\
\hline Sal & N/A & $\$ 250$ (unit) \\
\hline $\mathrm{T}$ & N/A & $24 \mathrm{t}$ \\
\hline $\mathrm{U}$ & N/A & $8 \mathrm{t}$ \\
\hline A & N/A & 100,000 \\
\hline
\end{tabular}

Table 6 Admission decision for scenario 1.

\begin{tabular}{|c|c|c|c|c|c|}
\hline Category & Optimum decision & $\beta=0.0005$ & $\beta=0.001$ & $\beta=0.002$ & $\beta=0.003$ \\
\hline \multirow[t]{2}{*}{1} & Quantity & 33,333 & 33,333 & 23,792 & 15,690 \\
\hline & Percentage & $100 \%$ & $100 \%$ & $71.4 \%$ & $47.1 \%$ \\
\hline \multirow[t]{2}{*}{2} & Quantity & 33,333 & 28,200 & 14,168 & 9600 \\
\hline & Percentage & $100 \%$ & $84.6 \%$ & $42.5 \%$ & $28.8 \%$ \\
\hline
\end{tabular}


Table 7 Admission decision for scenario 2 .

\begin{tabular}{|c|c|c|c|c|c|}
\hline Category & Optimum decision & $\beta=0.0005$ & $\beta=0.001$ & $\beta=0.002$ & $\beta=0.003$ \\
\hline \multirow[t]{2}{*}{1} & Quantity & 33,333 & 28,200 & 14,168 & 9600 \\
\hline & Percentage & $100 \%$ & $84.6 \%$ & $42.5 \%$ & $28.8 \%$ \\
\hline \multirow[t]{2}{*}{2} & Quantity & 33,333 & 28,200 & 14,168 & 9600 \\
\hline & Percentage & $100 \%$ & $84.6 \%$ & $42.5 \%$ & $28.8 \%$ \\
\hline \multirow[t]{2}{*}{3} & Quantity & 33,333 & 28,200 & 14,168 & 9600 \\
\hline & Percentage & $100 \%$ & $84.6 \%$ & $42.5 \%$ & $28.8 \%$ \\
\hline
\end{tabular}

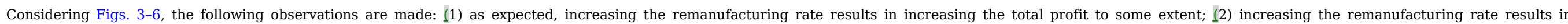

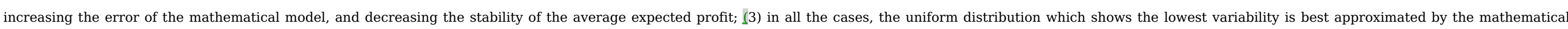

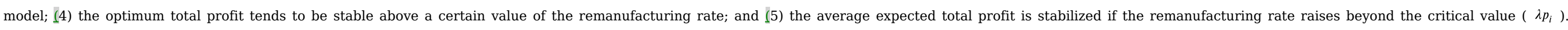

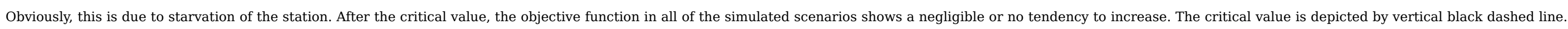

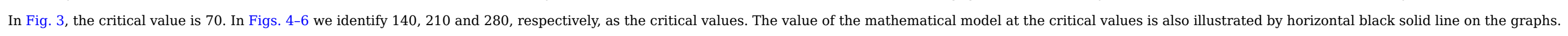

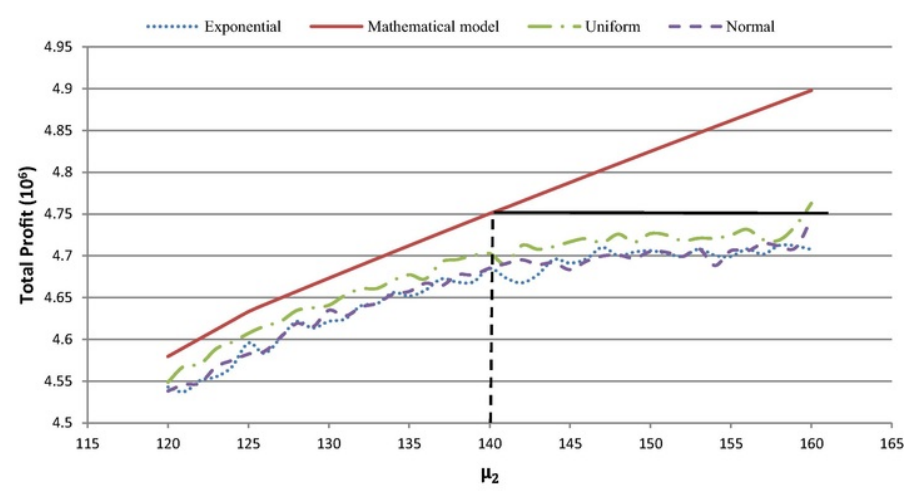

Fig. 4 Impact of the second station's remanufacturing rate, $\mu_{2}$, on the objective function for $\mu_{1}=70, \mu_{3}=90, \mu_{4}=100$, and $\lambda=700$. 


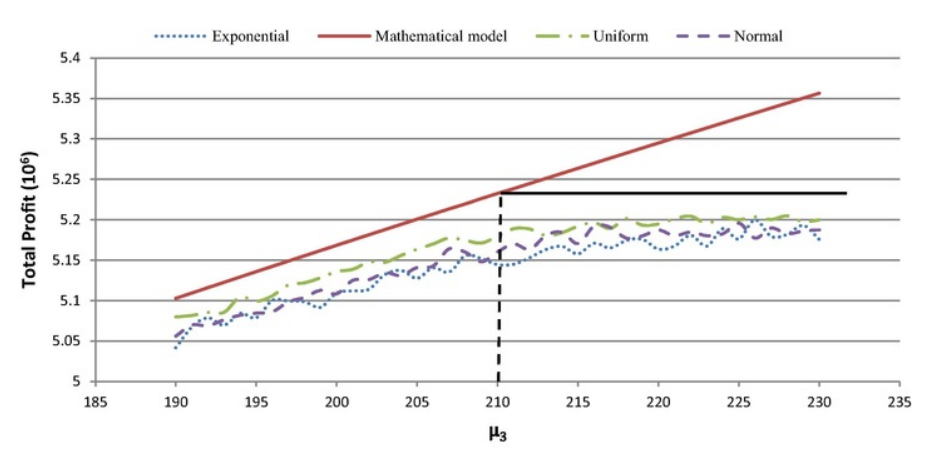

Fig. 5 Impact of the third station's remanufacturing rate, $\mu_{3}$, on the objective function for $\mu_{1}=70, \mu_{2}=80, \mu_{4}=100$, and $\lambda=700$

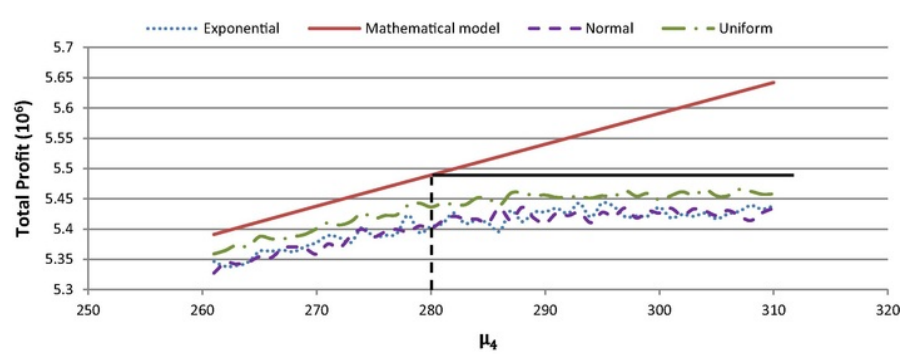

Fig. 6 Impact of the fourth station's remanufacturing rate, $\mu_{4}$, on the objective function $\mu_{1}=70, \mu_{2}=80, \mu_{3}=90, \lambda=700$.

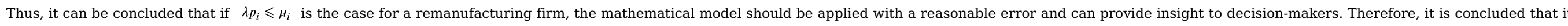
is also possible to apply this model where $\lambda p_{i} \leqslant \mu_{i}, \forall i \in I$.

In the next section, the sensitivity analysis is performed to investigate the effect of the model parameters.

\subsection{Sensitivity analysis of the key parameters for HP printers' case}

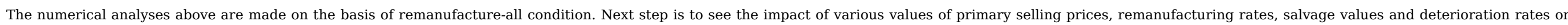

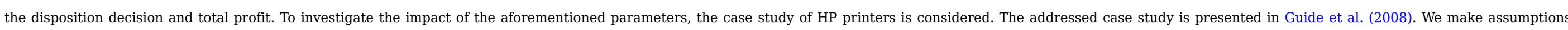

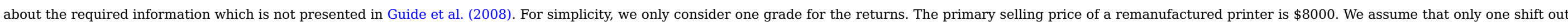

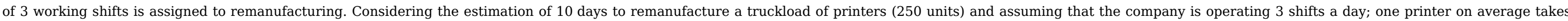

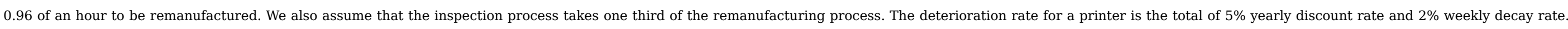

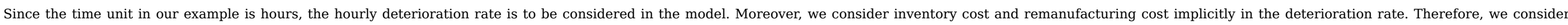

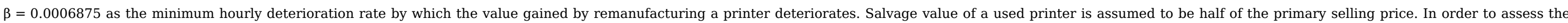

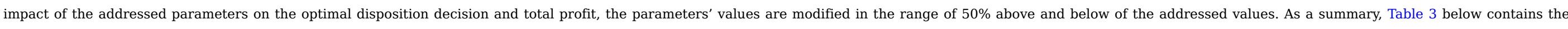
parameters considered in the numerical experiments.

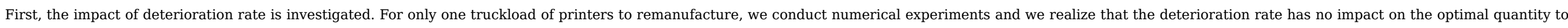
remanufacture. However, assuming that 2 full trucks deliver the used printers to the firm, the deterioration rate does affect the total profit and the optimal remanufacturing quantity, as illustrated in Fig. 7. 


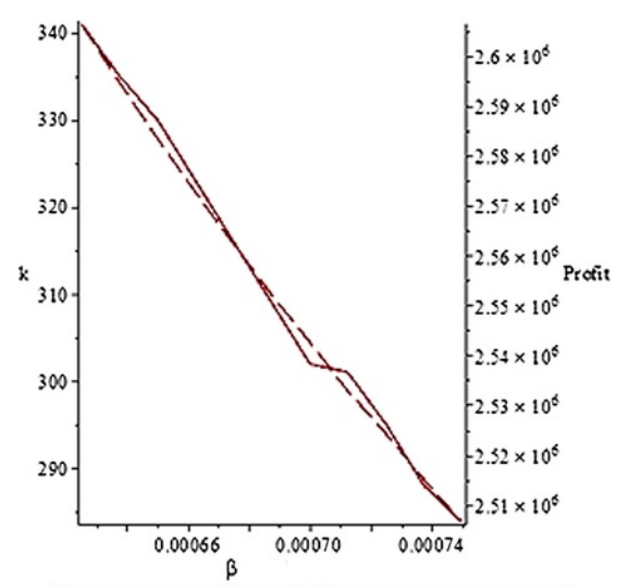

Remanufacturing quantity $(\mathrm{k})--$ Total profil

Fig. 7 Impact of the deterioration rate, $\beta$, on the optimal remanufacturing quantity and total profit for 2 truckloads of printers.

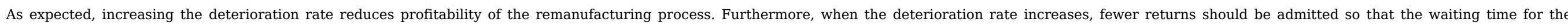

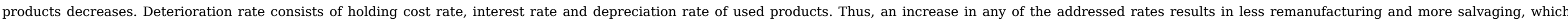
reduces the total profit.

In Fig. 8, the primary selling price, $r_{i}$ is graphed against the optimal remanufacturing quantity and the optimal total profit.

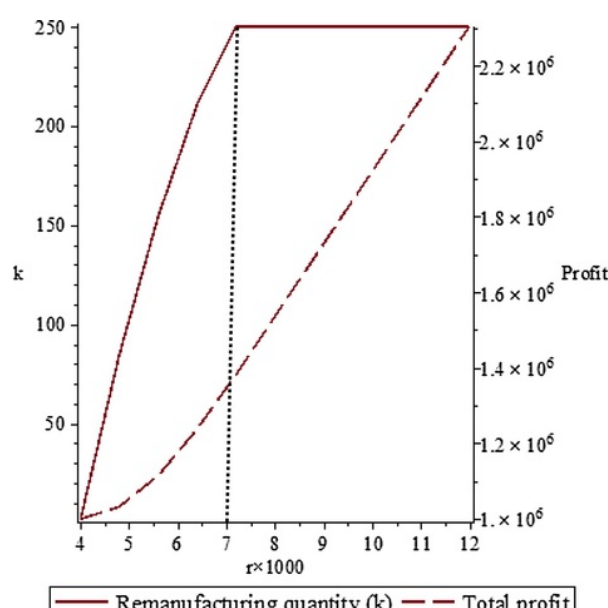

Fig. 8 Impact of the primary selling price, $r$, on the optimal remanufacturing quantity and total profit for one truckload or $k=250$ printers.

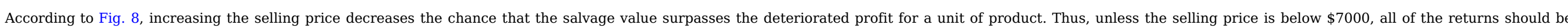

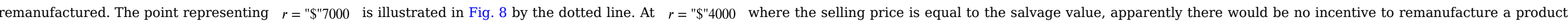
Both the remanufacturing quantity and total profit increase by increasing the selling price. Next, we aim at investigating the impact of Salvage value on the remanufacturing quantity and the total profit.

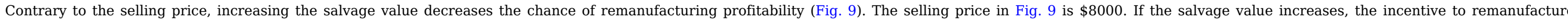

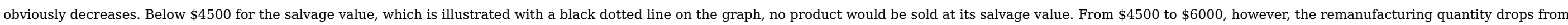




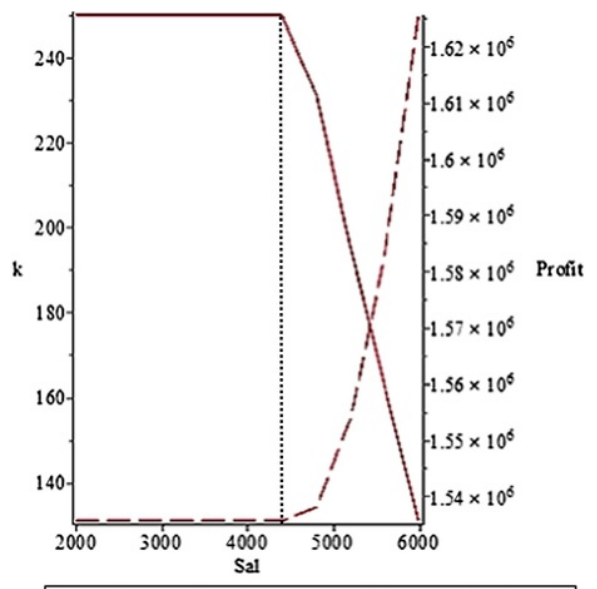

Fig. 9 Impact of the salvage value, Sal, on the optimal remanufacturing quantity and the total profit for one truckload of $k=250$ printers.

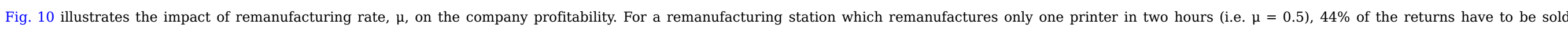

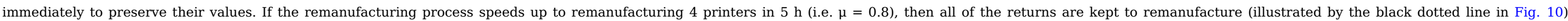
Increasing the remanufacturing rate, $\mu$, definitely increases the profit, as all of the returns would be remanufactured and sold in a relatively shorter time, comparing with the salvage value.

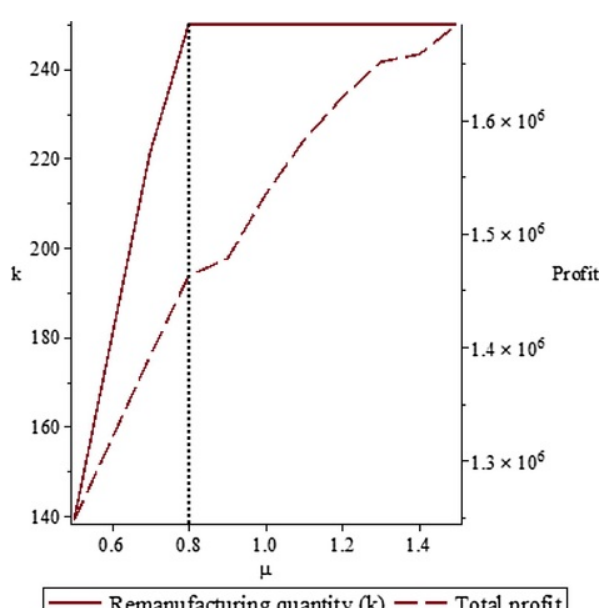

$$
\text { - Remanufacturing quantity }(\mathrm{k})-- \text { Total profit }
$$

Fig. 10 Impact of the process rate (number of printers to remanufacture per $t$ ) on the optimal remanufacturing quantity and total profit.

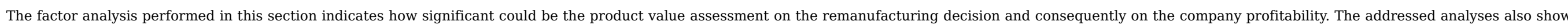
the drastic impact of remanufacturing process acceleration on the total profit.

\subsection{Additional numerical analysis}

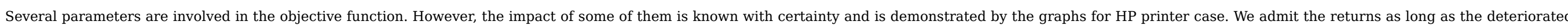




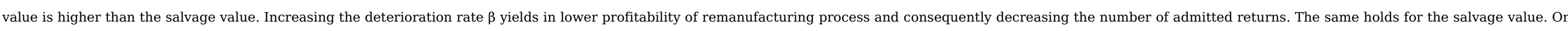

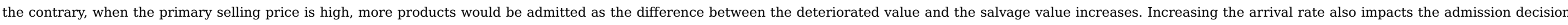

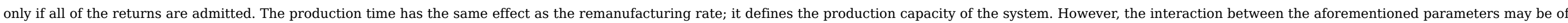

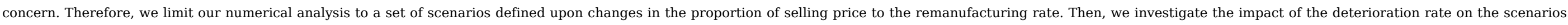

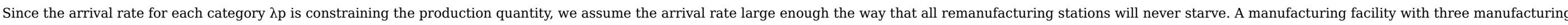
stations is considered. Five different scenarios are taken into account for the combination of primary selling prices and remanufacturing rates. The addressed scenarios are introduced in Table 4 below:

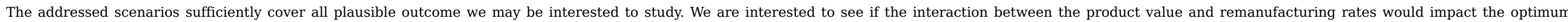

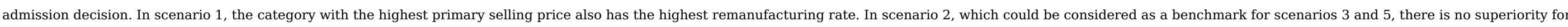

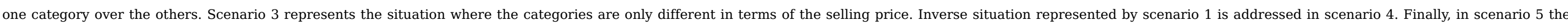

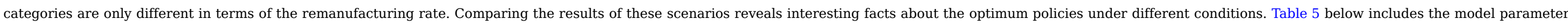
values in the numerical study.

For each scenario, the quantity and percentage of admitted returns are provided in Tables 6-10.

Table 8 Admission decision for scenario 3 .

\begin{tabular}{|c|c|c|c|c|c|}
\hline Category & Optimum decision & $\beta=0.0005$ & $\beta=0.001$ & $\beta=0.002$ & $\beta=0.003$ \\
\hline \multirow[t]{2}{*}{1} & Quantity & 33,333 & 33,333 & 17,739 & 11,998 \\
\hline & Percentage & $100 \%$ & $100 \%$ & $53.2 \%$ & $36.0 \%$ \\
\hline \multirow[t]{2}{*}{2} & Quantity & 33,333 & 28,200 & 14,168 & 9600 \\
\hline & Percentage & $100 \%$ & $84.6 \%$ & $42.5 \%$ & $28.8 \%$ \\
\hline \multirow[t]{2}{*}{3} & Quantity & 33,333 & 18,000 & 9000 & 6108 \\
\hline & Percentage & $100 \%$ & $54.0 \%$ & $27.0 \%$ & $18.3 \%$ \\
\hline
\end{tabular}

Table 9 Admission decision for scenario 4 .

\begin{tabular}{|c|c|c|c|c|c|}
\hline Category & Optimum decision & $\beta=0.0005$ & $\beta=0.001$ & $\beta=0.002$ & $\beta=0.003$ \\
\hline \multirow[t]{2}{*}{1} & Quantity & 33,333 & 23,237 & 12,000 & 7882 \\
\hline & Percentage & $100 \%$ & $69.7 \%$ & $36.0 \%$ & $23.7 \%$ \\
\hline \multirow[t]{2}{*}{2} & Quantity & 33,333 & 28,200 & 14,168 & 9600 \\
\hline & Percentage & $100 \%$ & $84.6 \%$ & $42.5 \%$ & $28.8 \%$ \\
\hline \multirow[t]{2}{*}{3} & Quantity & 33,333 & 23,600 & 11,953 & 8000 \\
\hline & Percentage & $100 \%$ & $70.8 \%$ & $35.8 \%$ & $24.0 \%$ \\
\hline
\end{tabular}

Table 10 Admission decision for scenario 5. 


\begin{tabular}{|c|c|c|c|c|c|}
\hline Category & Optimum decision & $\beta=0.0005$ & $\beta=0.001$ & $\beta=0.002$ & $\beta=0.003$ \\
\hline \multirow[t]{2}{*}{1} & Quantity & 33,333 & 18,799 & 9200 & 6262 \\
\hline & Percentage & $100 \%$ & $56.4 \%$ & $27.6 \%$ & $18.8 \%$ \\
\hline \multirow[t]{2}{*}{2} & Quantity & 33,333 & 28,200 & 14,382 & 9600 \\
\hline & Percentage & $100 \%$ & $84.6 \%$ & $43.1 \%$ & $28.8 \%$ \\
\hline \multirow[t]{2}{*}{3} & Quantity & 33,333 & 33,333 & 18,890 & 12,257 \\
\hline & Percentage & $100 \%$ & $100 \%$ & $56.6 \%$ & $36.8 \%$ \\
\hline
\end{tabular}

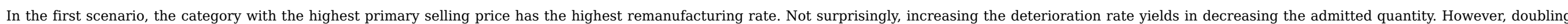
the deterioration rate had more significant effect on the categories with lower selling prices and remanufacturing rates.

In scenario 2, all categories have the same selling price and remanufacturing rate. These results could be considered as benchmark for scenarios 3 and 5 .

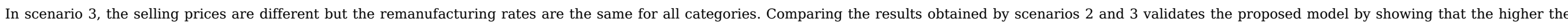
primary selling price and the remanufacturing rate are, the more of the returns should be admitted.

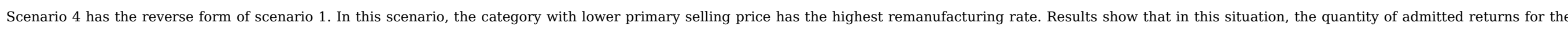
category with medium primary selling price and remanufacturing rate is more than the two other categories. Moreover, the admitted quantity for categories 1 and 3 is happened to be almost the same.

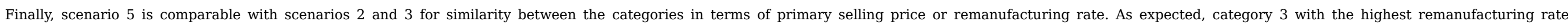
experienced the lowest reduction in admission quantity.

The admitted proportions for all scenarios are illustrated in Figs. 11-15 presented below.

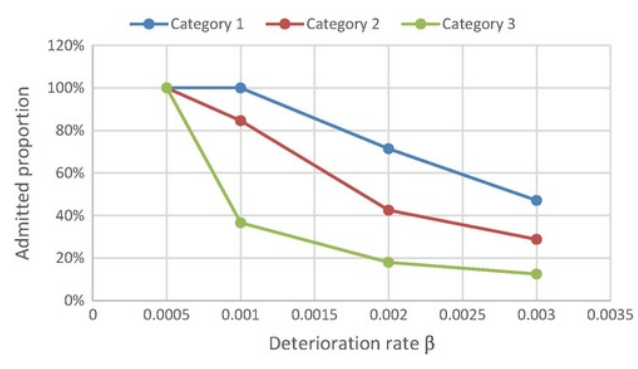

Fig. 11 Admitted proportions for scenario 1.

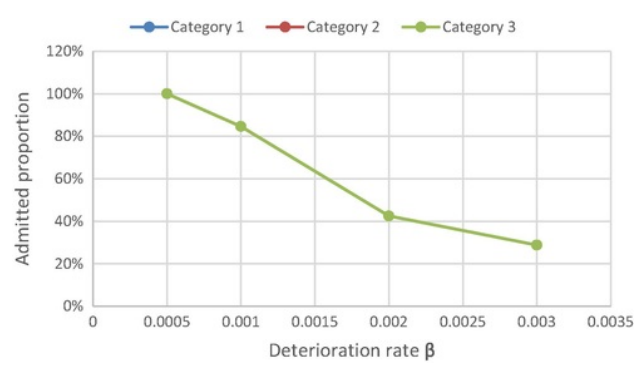


Fig. 12 Admitted proportions for scenario 2.

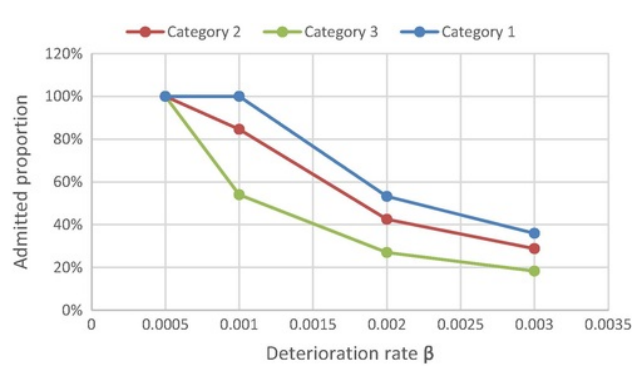

Fig. 13 Admitted proportions for scenario 3.

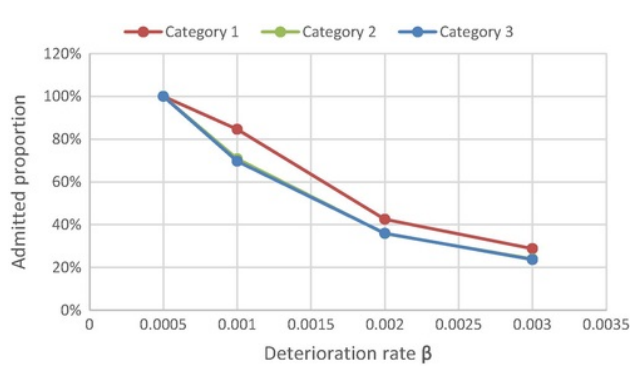

Fig. 14 Admitted proportions for scenario 4.

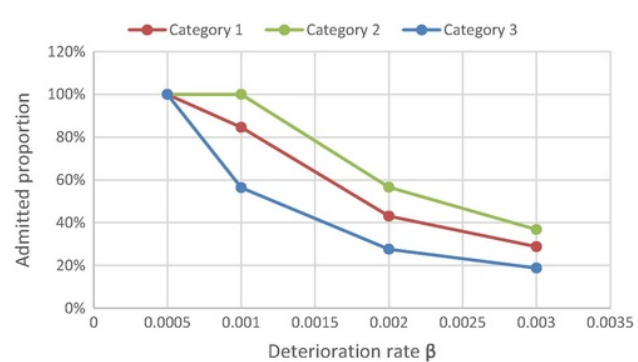

Fig. 15 Admitted proportions for scenario 5.

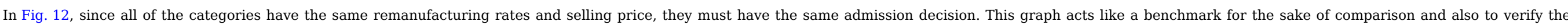
model.

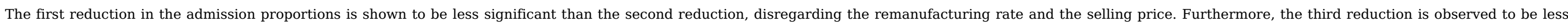

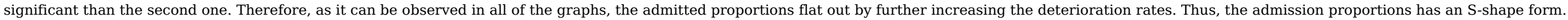

\section{Conclusion}

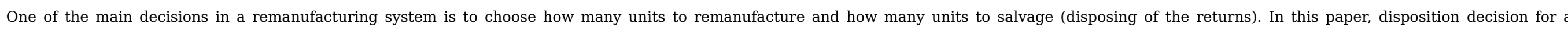

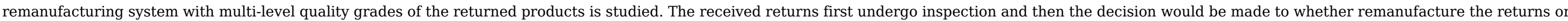
sell them immediately at their salvage value. A mathematical model is presented to approximate the total profit.

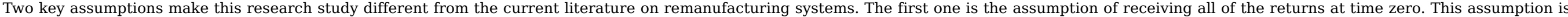

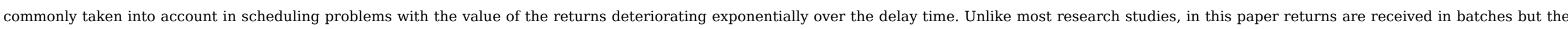




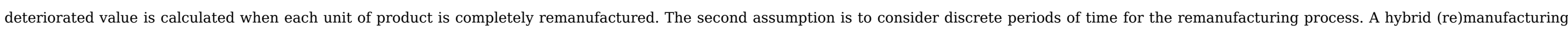

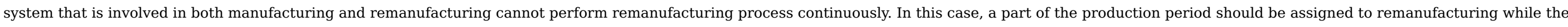

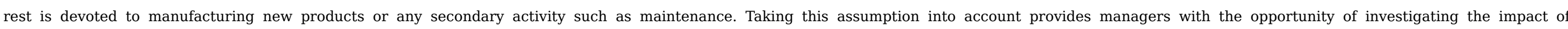
increasing/decreasing the operating period length for remanufacturing activities on the profitability of the company.

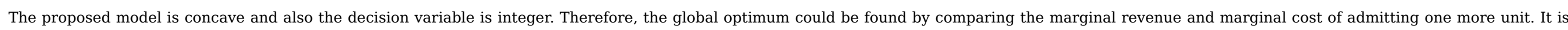
emphasized that since the objective function is not continuous and also the decision space is integer, the closed form of the optimum decision is not of interest.

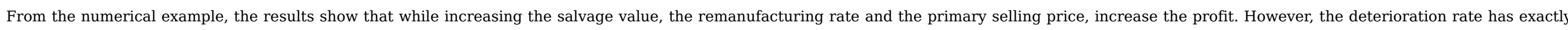

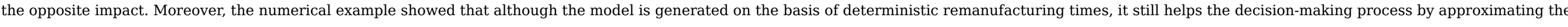

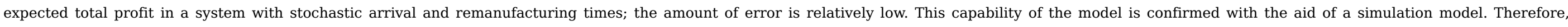
decision makers may use this model even if the system under investigation is stochastic.

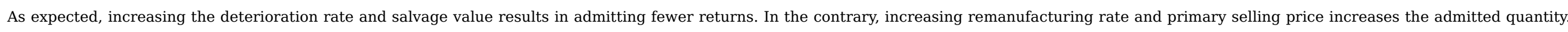

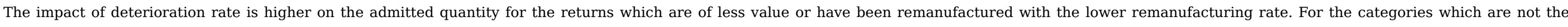

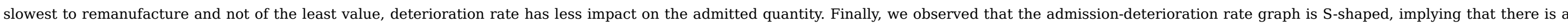
unique interval where each category undergoes faster decline in the admitted proportion.

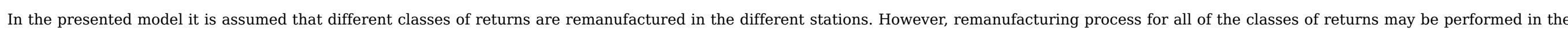

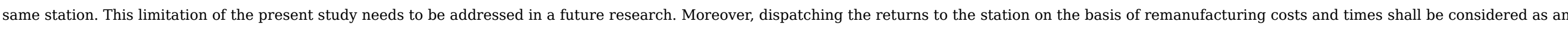
extension of this paper.

\section{Appendix A}

To obtain Eqs. (6)-(10), we have to solve the following series:

$$
\sum_{k=0}^{k=n} a r^{k}=a \frac{1-r^{k+1}}{1-r}
$$

In Eq. (6) we have:

$$
\sum_{n=1}^{n=S_{i}} r_{i} e^{-\beta\left(\frac{1}{\lambda p_{i}}+\frac{n}{\mu_{i}}\right)}=\sum_{n=1}^{n=S_{i}} r_{i} e^{-\beta\left(\frac{1}{\lambda p_{i}}\right)}\left(e^{-\beta\left(\frac{n}{\mu_{i}}\right)}\right)=\sum_{n=1}^{n=S_{i}} r_{i} e^{-\beta\left(\frac{1}{\lambda p_{i}}\right)}\left(e^{-\left(\frac{\beta}{\mu_{i}}\right)}\right)^{n}
$$

where $r_{i} e^{-\beta\left(\frac{1}{\lambda p_{i}}\right)}$ is the fixed part ( $a$ in A.1) and $e^{-\beta\left(\frac{1}{\mu_{i}}\right)}$ is the terms whose power changes ( $r$ in A.1). Note that (A.1) starts from $k=0$. Starting from $k=1$ we have:

$$
\sum_{k=1}^{k=n} a r^{k}=a \frac{1-r^{k+1}}{1-r}-a=a\left(\frac{1-r^{k+1}}{1-r}-1\right)=a\left(\frac{r-r^{k+1}}{1-r}\right)=a r\left(\frac{1-r^{k}}{1-r}\right)
$$

Using (A.3), the Eq. (A.2) would be:

$$
\sum_{n=1}^{n=S_{i}} r_{i} e^{-\beta\left(\frac{1}{\lambda p_{i}}\right)}\left(e^{-\left(\frac{\beta}{\mu_{i}}\right)}\right)^{n}=r_{i} e^{-\beta\left(\frac{1}{\lambda p_{i}}+\frac{1}{\mu_{i}}\right)}\left(\frac{1-\left(e^{-\left(\frac{\beta}{\mu_{i}}\right)}\right)^{S_{i}}}{1-\left(e^{-\left(\frac{\beta}{\mu_{i}}\right)}\right)}\right)=r_{i} e^{-\beta\left(\frac{1}{\lambda p_{i}}+\frac{1}{\mu_{i}}\right)}\left(\frac{1-e^{-\frac{S_{i} \beta}{\mu_{i}}}}{1-e^{-\frac{\beta}{\mu_{i}}}}\right)
$$

which is the same as (6).

In a similar way, for Eq. (7) we have: 


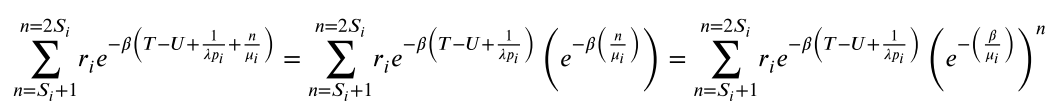

where $r_{i} e^{-\beta\left(T-U+\frac{1}{\lambda p_{i}}\right)}$ is the fixed part ( $a$ in A.1) and $e^{-\beta\left(\frac{1}{\mu_{i}}\right)}$ is the terms whose power changes ( $r$ in A.1). However, we make the following transformation:

$$
\sum_{n=S_{i}+1}^{n=2 S_{i}} r_{i} e^{-\beta\left(T-U+\frac{1}{\lambda p_{i}}\right)}\left(e^{-\left(\frac{\beta}{\mu_{i}}\right)}\right)^{n}=\sum_{n=1}^{n=S_{i}} r_{i} e^{-\beta\left(T-U+\frac{1}{\lambda p_{i}}\right)}\left(e^{-\left(\frac{\beta}{\mu_{i}}\right)}\right)^{n+S_{i}}=\sum_{n=1}^{n=S_{i}} r_{i} e^{-\beta\left(T-U+\frac{1}{\lambda p_{i}}\right)}\left(e^{-\left(\frac{\beta}{\mu_{i}}\right)}\right)^{S_{i}}\left(e^{-\left(\frac{\beta}{\mu_{i}}\right)}\right)^{n}
$$

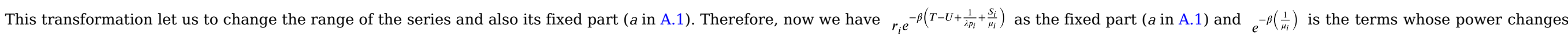
$(r$ in A.1).

Using (A.3) to solve (A.6) we have:

$$
\left.\sum_{n=1}^{n=S_{i}} r_{i} e^{-\beta\left(T-U+\frac{1}{\lambda_{i}}+\frac{S_{i}}{\mu}\right)}\left(e^{-\left(\frac{\beta}{\mu_{i}}\right)}\right)^{n}=r_{i} e^{-\beta\left(T-U+\frac{1}{\lambda p_{i}}+\frac{S_{i}}{\mu_{i}}\right.}\right)\left(e^{-\beta \frac{1}{\mu_{i}}}\right)\left(\frac{1-e^{-\frac{S_{i} \beta}{\mu_{i}}}}{1-e^{-\frac{\beta}{\mu_{i}}}}\right)=r_{i} e^{-\beta\left(T-U+\frac{1}{\lambda_{p_{i}}}+\frac{S_{i}+1}{\mu_{i}}\right)}\left(\frac{1-e^{-\frac{S_{i} \beta}{\mu_{i}}}}{1-e^{-\frac{\beta}{\mu_{i}}}}\right)
$$

which is the same as Eq. (7).

If we go through the same calculations for the third period, we will obtain:

$$
\left.\left.\sum_{n=1}^{n=S_{i}} r_{i} e^{-\beta\left(2(T-U)+\frac{1}{\lambda p_{i}}+\frac{2 S_{i}}{\mu}\right.}\right)\left(e^{-\left(\frac{\beta}{\mu_{i}}\right)}\right)^{n}=r_{i} e^{-\beta\left(2(T-U)+\frac{1}{\lambda p_{i}}+\frac{2 S_{i}}{\mu_{i}}\right)}\left(e^{-\beta \frac{1}{\mu_{i}}}\right)\left(\frac{1-e^{-\frac{S_{i} \beta}{\mu_{i}}}}{1-e^{-\frac{\beta}{\mu_{i}}}}\right)=r_{i} e^{-\beta\left(2(T-U)+\frac{1}{\lambda p_{i}}+\frac{2 S_{i}+1}{\mu_{i}}\right.}\right)\left(\frac{1-e^{-\frac{S_{i} \beta}{\mu_{i}}}}{1-e^{-\frac{\beta}{\mu_{i}}}}\right)
$$

Looking at the trend in (A.4), (A.7) and (A.8), we conclude that for the Rth period we have:

$$
\left.r_{i} e^{-\beta\left((R-1)(T-U)+\frac{1}{\lambda p_{i}}+\frac{(R-1) S_{i}+1}{\mu_{i}}\right.}\right)\left(\frac{1-e^{-\frac{S_{i} \beta}{\mu_{i}}}}{1-e^{-\frac{\beta}{\mu_{i}}}}\right)
$$

Therefore, the total deteriorated profit from the first period to the Rth period is:

$$
\left.\sum_{n=1}^{n=R} r_{i} e^{-\beta\left((n-1)(T-U)+\frac{1}{\lambda p_{i}}+\frac{(n-1) S_{i}+1}{\mu_{i}}\right.}\right)\left(\frac{1-e^{-\frac{S_{i} \beta}{\mu_{i}}}}{1-e^{-\frac{\beta}{\mu_{i}}}}\right)
$$

Eq. (A.10) is transformed to:

$$
\sum_{n=1}^{n=R} r_{i} e^{-\beta\left(\frac{1}{\lambda p_{i}}+\frac{1}{\mu_{i}}\right)}\left(\frac{1-e^{-\frac{S_{i} \beta}{\mu_{i}}}}{1-e^{-\frac{\beta}{\mu_{i}}}}\right)\left(e^{-\beta\left((n-1)(T-U)+\frac{(n-1) S_{i}}{\mu_{i}}\right)}\right)=\sum_{n=1}^{n=R} r_{i} e^{-\beta\left(\frac{1}{\lambda p_{i}}+\frac{1}{\mu_{i}}\right)}\left(\frac{1-e^{-\frac{S_{i} \beta}{\mu_{i}}}}{1-e^{-\frac{\beta}{\mu_{i}}}}\right)\left(e^{-\beta\left((T-U)+\frac{S_{i}}{\mu_{i}}\right)}\right)^{n-1}=\sum_{n=0}^{n=R-1} r_{i} e^{-\beta\left(\frac{1}{\lambda p_{i}}+\frac{1}{\mu_{i}}\right)}\left(\frac{1-e^{-\frac{S_{i} \beta}{\mu_{i}}}}{1-e^{-\frac{\beta}{\mu_{i}}}}\right)\left(e^{-\beta\left((T-U)+\frac{S_{i}}{\mu_{i}}\right)}\right)^{n}
$$

where $r_{i} e^{-\beta\left(\frac{1}{\lambda p_{i}}+\frac{1}{\mu_{i}}\right)}\left(\frac{1-e^{-\frac{S_{i} \beta}{\mu_{i}}}}{1-e^{-\frac{\beta}{\mu_{i}}}}\right)$ is the fixed part and $\left(e^{-\beta\left((T-U)+\frac{S_{i}}{\mu_{i}}\right)}\right)$ is the part whose power changes. Since the range of the series has changed, we cannot use (A.3). Using we have:

$$
\sum_{n=0}^{n=R-1} r_{i} e^{-\beta\left(\frac{1}{\lambda p_{i}}+\frac{1}{\mu_{i}}\right)}\left(\frac{1-e^{-\frac{S_{i} \beta}{\mu_{i}}}}{1-e^{-\frac{\beta}{\mu_{i}}}}\right)\left(e^{-\beta\left((T-U)+\frac{S_{i}}{\mu_{i}}\right)}\right)^{n}=r_{i} e^{-\beta\left(\frac{1}{\lambda_{p}}+\frac{1}{\mu_{i}}\right)}\left(\frac{1-e^{-\beta \frac{S_{i}}{\mu_{i}}}}{1-e^{-\beta \frac{1}{\mu_{i}}}}\right)\left(\frac{\left.1-e^{-\beta\left(T-U+\frac{S_{i}}{\mu_{i}}\right.}\right)^{R-1}}{\left.1-e^{-\beta\left(T-U+\frac{S_{i}}{\mu_{i}}\right.}\right)}\right)=r_{i} e^{-\beta\left(\frac{1}{\lambda_{p_{i}}}+\frac{1}{\mu_{i}}\right)}\left(\frac{1-e^{-\beta \frac{S_{i}}{\mu_{i}}}}{1-e^{-\beta \frac{1}{\mu_{i}}}}\right)\left(\frac{1-e^{-\beta(R-1)\left(T-U+\frac{S_{i}}{\mu_{i}}\right)}}{\left.1-e^{-\beta\left(T-U+\frac{S_{i}}{\mu_{i}}\right.}\right)}\right)
$$

which is the same as (9).

Eq. (10) can be obtained in the same way as (6)-(8). and Engineering Council of Canada (NSERC) under the Discovery Grant Program.References 
Alidaee B. and Womer N.K., Scheduling with time dependent processing times: Review and extensions, The Journal of the Operational Research Society 50 (7), $1999,711-720$.

Blackburn J.D., Guide V.D.R., Souza G.C. and Van Wassenhove L.N., Reverse supply chains for commercial returns, California Management Review 46 (2), $2004,6-22$.

Denizel M., Ferguson M. and Souza G., Multiperiod remanufacturing planning with uncertain quality of inputs, IEEE Transactions on Engineering Management 57 (3), 2010, 394-404,

http://dx.doi.org/10.1109/TEM.2009.2024506.

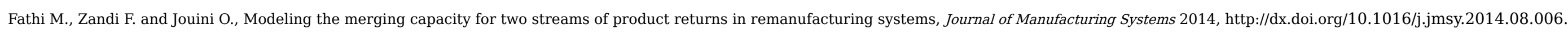

Ferguson M.E., Fleischmann M. and Souza G.C., A profit-maximizing approach to disposition decisions for product returns, Decision Sciences 42 (3), 2011 , 773-798.

Ferrer G. and Swaminathan J.M., Managing new and remanufactured products, Management Science 52 (1), 2006, 15-26.

Galbreth M.R. and Blackburn J.D., Optimal acquisition quantities in remanufacturing with condition uncertainty, Production and Operations Management 19 (1), $2010,61-69$.

Geyer R., Van Wassenhove L.N. and Atasu A., The economics of remanufacturing under limited component durability and finite product life cycles, Management Science 53 (1), 2007 , 88-100.

Guide V.D.R., Jr., Production planning and control for remanufacturing: Industry practice and research needs, Journal of Operations Management 18 (4), $2000,467-483$.

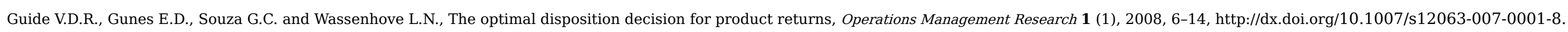

Harrison J.M., Dynamic scheduling of a multiclass queue: Discount optimality, Operations Research 23 (2), 1975, 270-282.

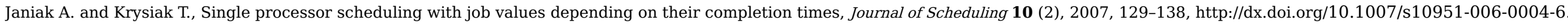

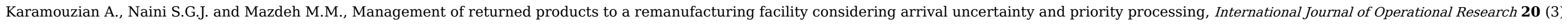
2014, 331, http://dx.doi.org/10.1504/IJOR.2014.062454.

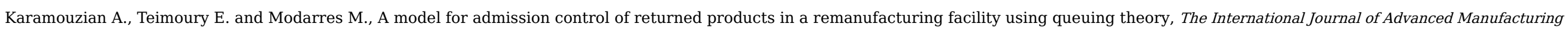
Technology 54 (1-4), 2011, 403-412, http://dx.doi.org/10.1007/s00170-010-2933-7.

Lund R., Remanufacturing: An American resource, In: Proceedings of the fifth international congress environmentally conscious design and manufacturing Vol. 16, 1998, June.

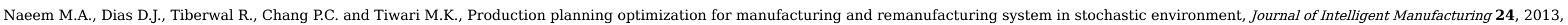
$717-728$.

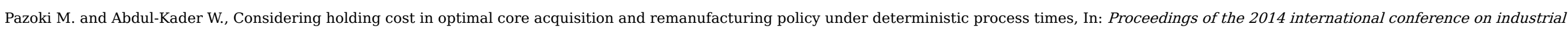
engineering and operations management, 2014, Bali; Indonesia, 2371-2379.

Souza G.C., Ketzenberg M.E. and Guide V.D.R., Capacitated remanufacturing with service level constraints, Production and Operations Management 11 (2), 2002 , 231-248.

Su C. and Xu A., Buffer allocation for hybrid manufacturing/remanufacturing system considering quality grading, International Journal of Production Research 52 (5), 2014, 1269-1284, http://dx.doi.org/10.1080/00207543.2013.828165.

Teunter R.H. and Flapper S.D.P., Optimal core acquisition and remanufacturing policies under uncertain core quality fractions, European Journal of Operational Research 210 (2), 2011, 241-248,

http://dx.doi.org/10.1016/j.ejor.2010.06.015.

Voutsinas T.G. and Pappis C.P., Scheduling jobs with values exponentially deteriorating over time, International Journal of Production Economics 79, $2002,163-169$.

Voutsinas T.G. and Pappis C.P., A branch and bound algorithm for single machine scheduling with deteriorating values of jobs, Mathematical and Computer Modelling 52 (1-2), 2010, 55-61,

http://dx.doi.org/10.1016/j.mcm.2009.12.024

\section{Footnotes}


1“Remanufactured goods: An overview of the U.S. and global industries, markets, and trades.” US International Trade Commission, USITS publication, Oct 2012.

4

Highlights

- Optimal disposition.

- Quantity to remanufacture.

- Quantity to salvage.

- Deterioration value for returns and time value of the products.

\section{Queries and Answers}

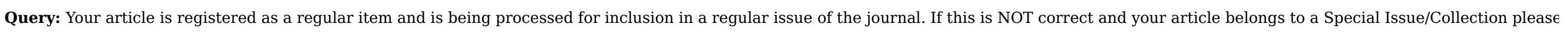
contact v.pandey.1@elsevier.com immediately prior to returning your corrections.

Answer: Yes; this article is registered as a regular item and is being processed for inclusion in a regular issue of the journal.

Query: The author names have been tagged as given names and surnames (surnames are highlighted in teal color). Please confirm if they have been identified correctly. Answer: yes

Query: Please check the edits made in the sentence 'The total remanufacturing profit function...', and correct if necessary.

Answer: Yes 